\title{
From Womb to Graveyard: Blockchain in Healthcare
}

\author{
Debajani Mohanty ${ }^{1}$ and Rajul Rastogi*2 \\ ${ }^{1}$ Senior Technical Architect, NIIT Technologies Limited, India \\ ${ }^{2}$ Department of Radiodiagnosis, Associate Professor, Teerthanker Mahaveer Medical College \& Research Center, India
}

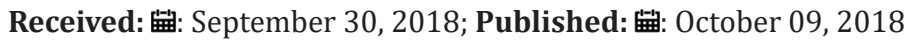

*Corresponding author: Rajul Rastogi, Department of Radiodiagnosis, Teerthanker Mahaveer Medical College \& Research Center, India

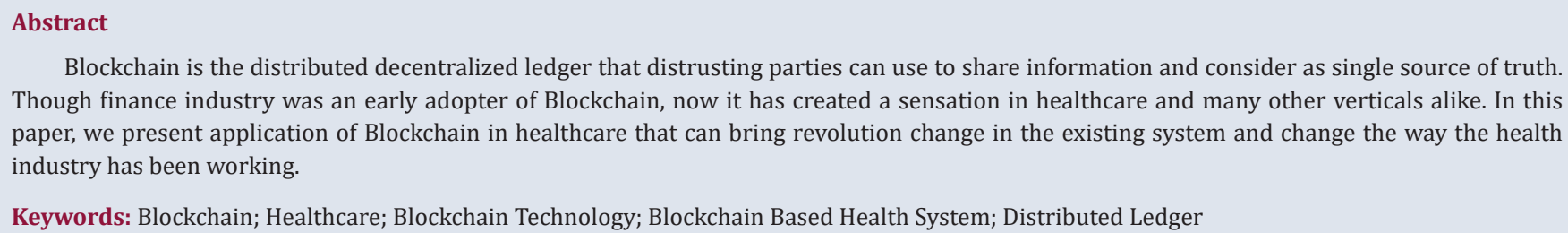

Blockchain is the distributed decentralized ledger that distrusting parties can use to share information and consider as single source of truth. Though finance industry was an early adopter of Blockchain, now it has created a sensation in healthcare and many other verticals alike. In this paper, we present application of Blockchain in healthcare that can bring revolution change in the existing system and change the way the health industry has been working.

Keywords: Blockchain; Healthcare; Blockchain Technology; Blockchain Based Health System; Distributed Ledger

\section{Introduction}

Blockchain technology has brought the biggest disruption to almost all industries in 21st century. As per a report by International Data Corporation (IDC), worldwide spending in this emerging technology is growing at an unbelievable rate of $81.2 \%$ and would reach $\$ 9.7$ Billion by 2021 [1]. Blockchain though started with and often associated with crypto currencies, yet has touched almost every industry and in the process has transform the way we used to work before. Blockchain is a publicly or privately available distributed digital ledger that mutually untrusting parties can use for business transactions and can refer to as the single source of truth. Unlike traditional databases, Blockchain ledger works in append-only mode. Also for its decentralized nature it has no single point of failure. Thus it's a revolutionary new way for complex business transactions between organizations resulting in real-time settlements, in a process cutting down on time, cost and efforts by middlemen and thus improving transparency and trust of the overall ecosystem. When it comes to healthcare, Blockchain is projected to be $\$ 176.8$ million in 2018 , and is estimated to grow over $\$ 5.61$ billion by the end of 2025 [2].

\section{Applications of Blockchain in Healthcare}

Few of the very promising Blockchain use cases in healthcare domain are as below
a) Provenance of Medicine,
b) Medical insurance processing,
c) Global health governance.

\section{Provenance \& Tracking in Medicine}

Provenance of medicine or any other resource is all about tracking of its origin and history. Let's consider a scenario where a particular medicine is manufactured, stored, transported to hospitals or stores. Expensive drugs like the ones used in chemotherapy are mandated to be kept at a certain temperature; any breach may cause a loss in their effectiveness.

Issues with traditional mechanism of medicine supply-chain

a) Lack of common platform for sharing of information leading to wastage as well as inefficient supply,

b) All the information printed on the medicine as expiry date, manufacturing date, laboratory etc. has little traceability,

c) Error-prone record-keeping.

Such expensive medicines can be attached to temperature and location controlled Internet of Things (IoT) sensors that can feed environmental data continuously to the Blockchain ledger. The data on Blockchain can be stored in association with the barcode of the medicine which the customer can use to track all the important information related to the medicine and breach of contract if that has ever occurred.

Some of the advantages are as below:

a) Transparency results in trust throughout the supply chain,

b) Vendors and consumers know what they're getting and what they're paying for.

\section{Medical Insurance Processing}

IDC report claims that medical insurance claims automation is top-use case ofmanagement through Blockchain. Secureinformation sharing methods, which allow both healthcare providers and their covered entities to verify the accuracy of data, are crucial for ensuring 
proper medical services. This is where Blockchain becomes useful, as one of the major advantages of Blockchain is data integrity. A consortium based engagement between multiple parties including the insured, claimant, carrier, and any other third parties including service provider etc. will enable more reliable, foolproof and faster claims settlement process. Blockchain, with smart contracts, can simplify this currently cumbersome process, and thus provide an improved customer experience. Smart contracts can also enable auto-initiation, and in some cases settlement of claims too without much manual intervention. Blockchain will be very beneficial when insurance is combined with other services such as Death Claim, Health Claims etc.

Some of the advantages are as below

a. Automation saves time and operating costs,

b. Single source of truth for all parties to track data in real time.

\section{Global Health Governance \& Provenance}

During critical illness, a patient is often referred to visit tertiary care hospital by primary or secondary healthcare center for further diagnosis or treatment that needs sharing of patient's reports, which is often a cumbersome process. With Blockchain all data related to a patient can be stored on a distributed ledger. Hospitals and insurance organizations can get access to this data as single source of truth after getting consent from the patient and can do further study to diagnose diseases. Also, research labs can use this data for tracking source of a disease or even outbreak of a disease from early symptoms. As per a recent IDC report, by 2020, 1-in5 Healthcare organisations will adopt Blockchain [3]. According to Pushplata Patel, an analyst at BIS (Fullform) Research, "Around $14 \%$ of healthcare organizations are expected to have a Blockchain based system in place by the end of 2018 , while $70 \%$ are expected to have invested in getting the technology on-board by 2020" [4]. Other disruptive technologies as Artificial Intelligence (AI) and Robotics can work together with Blockchain, the decentralized economy to bring next generation healthcare to reality.

\section{Summary}

To summarize, Blockchain is an emerging technology in IT industry that has wide applications in healthcare and hence can revolutionize the existing healthcare system.

\section{References}

1. (2018) New IDC Spending Guide Sees Worldwide Blockchain Spending Growing to \$9.7 Billion in 2021.

2. (2018) Global Blockchain in Healthcare Market to Reach $\$ 5.61$ Billion by 2025, Reports BIS Research.

3. (2018) By 2020, 1-in-5 Healthcare organisations will adopt Blockchain says new IDC report.

4. (2018) Blockchain Market Projected to Grow Thanks to Technological Innovations.
ISSN: 2574-1241

DOI: 10.26717/BJSTR.2018.09.001840

Rajul Rastogi. Biomed J Sci \& Tech Res

This work is licensed under Creative Commons Attribution 4.0 License

Submission Link: https://biomedres.us/submit-manuscript.php

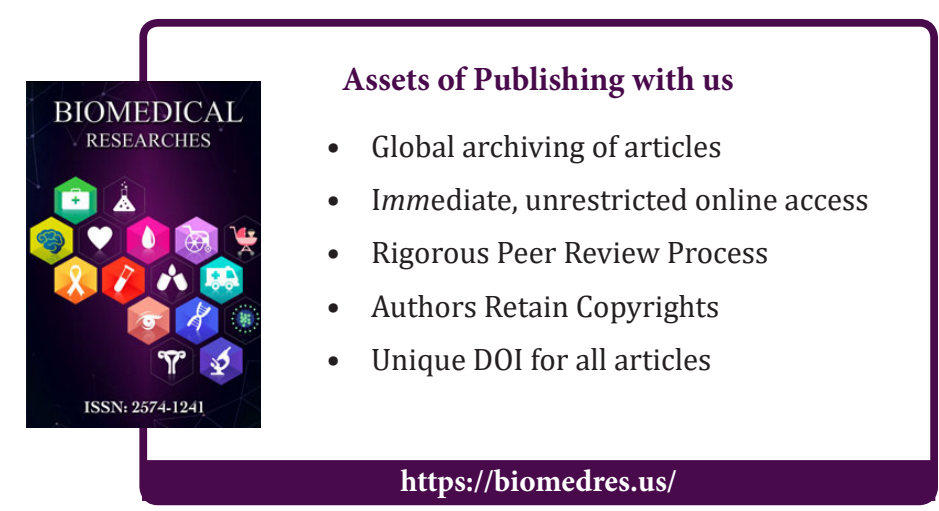

